\title{
Non-Destructive and Non-Contact Testing of Coatings
}

\author{
Bariska, Andor \\ Dr. Reinke, Nils \\ ZHAW School of Engineering \\ P.O. Box \\ $\mathrm{CH}-8401$ Winterthur
}

\section{Introduction}

Surfaces of airplanes, rail and road vehicles require multi-layered corrosion-protection coating, chipprotection coating and decorative coating optimised for their specific uses. Wear and tear of parts in motors, engines and industrial manufacturing plants can be reduced and their lifespan extended thanks to highly effective protective coatings. Many other items of our everyday life are coated for protective and decorative purposes.

The monitoring of the thickness of the coatings and other coating features during the production process (in-line monitoring) has, up to now, been complicated and costly. The most commonly methods used to test coatings are either contacting or require safety precautions because harmful radiation is emitted during the measuring procedure (laser, $x$-rays, beta-rays).

This lack of an easy-to-use in-line testing technology leads the coating industry to apply more coating material than.Thus there is a need for a reliable and easy-to-use testing system for industrial coating processes.

\section{Thermal Testing of Coatings}

We have developed a technology at the School of Engineering of the Zurich University of applied Sciences (ZHAW) that makes possible rapid, non-contact and non-destructive monitoring of multi-layered coatings on metal, ceramic, plastic and wood surfaces. The technology is based on the pulsed warming of the coating and the evaluation of the temperature progression.

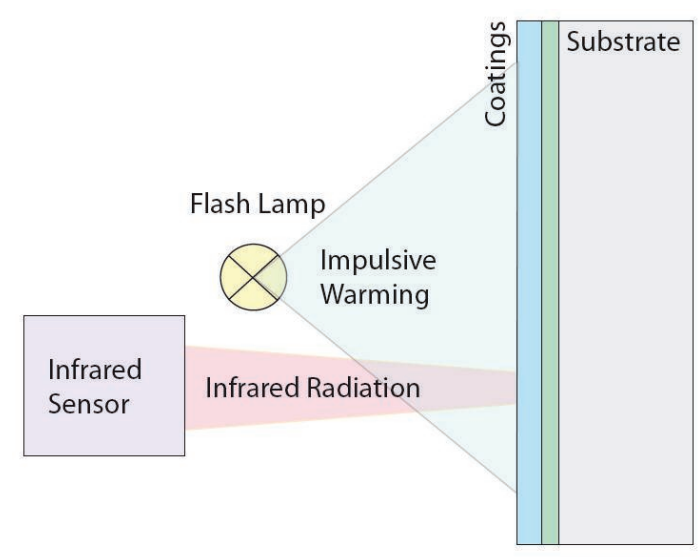

Fig. 1. Measurement Setup for Thermal Testing of Coatings.

The main components of the measurement system are a flash lamp and a high speed infrared sensor (see Fig. 1), [1]. The measurement process consists of the following steps:

1. The surface of the coating is warmed with the light flash. Typically, the surface temperature rises no more than a few degrees.

2. The light flash is converted into heat which immediately diffuses through the coating system, leaving a characteristic surface temperature time evolution, recorded using the high speed infrared sensor.

3. The heat diffusion is governed by the thermal properties of the coating and substrate materials as well as by the thickness and adhesion strength of the individual coating layers. Therefore using 
appropriate algorithms, these parameters can be deduced from the surface temperature time evolution.

The measuring system is robust and impervious to distance and tilting. It can repeat measurements every three seconds and is insensitive to humidity and temperature in the operating environment. The measurement spot size can be tuned to the application, standard size is $2 \mathrm{~mm}^{2}$.

\section{Benefits to Coating Industry}

The coating industry can benefit in various ways from products based on our technology. It is reliable and easy to use (including safe, fast, noncontact and nondestructive operation). We offer the following customer value:

1. Prevent warranty claims by detecting defective coatings, provide quality assurance and objective quality ratings.

2. Allowing rapid response to process deviations (reduce production down-time, avoiding costly repairs of defective goods).

3. Reduction of the running-in period necessary for new coating systems or materials.

4. Improved utilization of personnel (reduction of costs for quality control personnel).

5. Savings of coating material (lacquer, powder etc.).

6. Reduction of environmental footprint (wastewater contamination, $\mathrm{CO} 2$ emission, disposal of bio solids, improvement of the working conditions).

In the following, we give application examples of thermal testing of coatings.

\section{Testing of Thermal Barrier Coatings}

Thermal Barrier Coatings (TBC) consist of a ceramic top coat (typically zirconia) applied onto a bond coat to improve adhesion strength to the substrate (see Fig. 2). Their function are to protect e.g. gas turbine hot section components and combustion chambers of diesel engines, introducing a temperature gradient across the TBC of $100-300^{\circ} \mathrm{C}$ [2]. The thermal barrier effect is due to the low thermal conductivity of the ceramic material; a consequence of its porous nature. Therefore, important factors to determine during application of TBCs are the thickness and the porosity of the ceramic top coat.

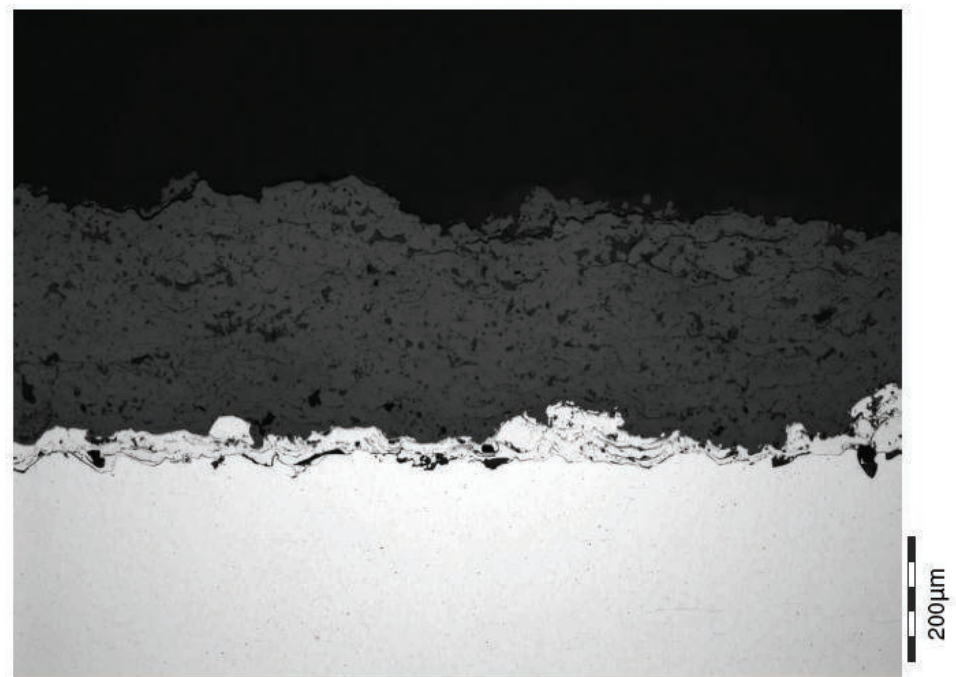

Fig. 2: Micrograph of a plasma sprayed thermal barrier coating coating on steel substrate (bottom), consisting of the bond coat (nickel alloy, middle) and the functional top coat (zirconia, top). (Source: Sulzer Metco, Wohlen)

Currently, the porosity of the ceramic top coat is tested using micrographing. Samples are taken from the production process and the porosity determined from microscope images taken from the prepared sample and analyzed in certified laboratories. This procedure results in a massive overhead for quality testing purposes due to lab costs and unused production time. 
On the other hand, our measurement system allows the simultaneous testing of both porosity and coating thickness in the production line and thus reduce the quality testing overhead.

\section{Relative Coating Thickness Measurement}

To illustrate the ease-of-use of our measurement system, we describe how to perform relative coating thickness measurement on unknown (i.e. uncalibrated) coatings and substrates. Often, the absolute coating thickness is not required and the knowledge of the relative coating thickness (with respect to a reference coating) already solves practical problems in the coating industry.

The procedure consists of two steps:

1. Reference Measurement. In this step, the surface temperature evolution after the light flash on a reference coating sample is recorded and stored as reference

2. Relative Coating Thickness Measurement. A second coating sample consisting of the same coating and substrate material combination, but different coating thickness, as the reference is tested. The surface temperature evolution on this sample after the light flash is also recorded. Using our proprietary algorithms, the relative thickness of the reference and test coatings can be determined from the reference and test measurements.

As can be seen from the above description, no calibration using additional measurement equipment is required for relative coating thickness measurement. Furthermore, it is also possible to store two reference measurements (e.g. for lower and upper coating thickness bounds) and evaluate a test coating compared with two references.

If the coating thickness of the reference coating is known, the absolute thickness of any coating consisting of the same coating material (on arbitrary substrates) can subsequently be determined without further calibration. This significantly reduces the calibration effort for our measurement systems.

\section{Conclusion}

We have presented an innovative measurement technology for the nondestructive and noncontact testing of coatings. The technology is based on warming the coating with a flash light and evaluating the resulting surface temperature evolution. It is reliable, easy-to-use and ready for in-line installation in industrial coating processes.

The main benefits arising from our technology for the coating industry are to save process time, to save coating material and to enable $100 \%$ quality control during the production process. This results in a more efficient and ecologically friendly coating process [3].

Winterthur Instruments $\mathrm{GmbH}$, a spin-off company from the ZHAW School of Engineering is responsible for the commercialization of this measurement technology.

\section{Links}

- www.engineering.zhaw.ch/schichtcharakterisierung

- www.youtube.com/watch?v=oK41PamlbjA

- $\quad$ www.winterthurinstruments.de

\section{References}

[1] N. Reinke, A. Bariska. Einfache Beschichtungskontrolle mit thermischer Schichtprüfung. Electrosuisse Bulletin SEV; October 2010.

[2] S. Ahmaniemi, Modified Thick Thermal Barrier Coatings. Dissertation, Tampere University of Technology, 2004.

[3] R. Krüser, Thermische Schichtprüfung: Oberflächen einfach kontrollieren, besser lackieren! 13, February 2011. 